\title{
Exponential Synchronization of Nonlinear Oscillators Under Sampled-Data Coupling
}

\author{
Jijju Thomas ${ }^{1}$, Erik Steur ${ }^{2}$, Christophe Fiter ${ }^{3}$, Laurentiu Hetel ${ }^{4}$, and Nathan van de Wouw ${ }^{5}$.
}

\begin{abstract}
This paper presents a novel approach towards synchronization analysis of nonlinear oscillatory systems, bidirectionally coupled via a networked communication channel. The system under consideration is a two-agent nonlinear system, under the constraint that information is transmitted between the two systems using a sampled-data communication strategy that could be periodic or aperiodic. The networked system dynamics is remodelled as a feedback-interconnection of a continuous-time system, and an operator that accounts for the communication constraints. By studying the properties of this feedback-interconnection in the framework of dissipativity theory, we provide a novel criterion that guarantees exponential synchronization. The provided criterion also aids in deciding the trade-off between a bound on the sampling intervals, the coupling gain, and the desired transient rate of synchronization. Finally, the theoretical results are illustrated using a two-agent Fitzhugh-Nagumo system.
\end{abstract}

\section{INTRODUCTION}

In many natural and engineering systems, the phenomenon of synchronization has been investigated by researchers and scientists from various fields. Typical examples of synchronization include flashing fireflies, firing neurons, cooperative control of multi-agent systems, etc. [1]. In control theory, synchronization of networked nonlinear oscillators is a topic that has specifically garnered attention owing to its significance in neural processes, communication systems, electronic circuits, etc. [2], [3], [4]. Such networked scenarios often result in individual sub-systems interacting bidirectionally. In comparison to weaker definitions of synchronization, i.e., in the form of consensus or synchronization to a set of equilibria, synchronization in the sense of asymptotic matching of time-varying (oscillatory) solutions, has been

This work was supported by project UCoCoS, funded by the European Union's EU Framework Programme for Research and Innovation, Horizon H2020, Grant Agreement No: 675080. The project was also funded by ANR Project ROCC-SYS (agreement ANR-14-CE27-0008)

${ }^{1}$ Jijju Thomas (j.thomas@tue.nl) is with Univ. Lille, CNRS, Inria, Centrale Lille, UMR 9189 - CRIStAL - Centre de Recherche en Informatique Signal et Automatique de Lille, F-59000 Lille, France, and the Department of Mechanical Engineering, Eindhoven University of Technology, 5600 MB, The Netherlands.

${ }^{2}$ Erik Steur (e.steur@tue.nl) is with the Department of Mechanical Engineering, and the Institute for Complex Molecular Systems, Eindhoven University of Technology, $5600 \mathrm{MB}$, The Netherlands.

${ }^{3}$ Christophe Fiter (christophe.fiter@univ-lille1.fr) is with Univ. Lille, CNRS, UMR 9189 - CRIStAL - Centre de Recherche en Informatique Signal et Automatique de Lille, F-59000 Lille, France.

${ }^{4}$ Laurentiu Hetel (laurentiu.hetel@ec-lille.fr) is with Univ. Lille, CNRS, Centrale Lille, UMR 9189 - CRIStAL - Centre de Recherche en Informatique Signal et Automatique de Lille, F-59000 Lille, France

${ }^{5}$ Nathan van de Wouw (n.v.d.wouw@tue.nl) is with the Department of Mechanical Engineering, Eindhoven University of Technology, 5600 MB, The Netherlands, and with the Department of Civil, Environmental and GeoEngineering, University of Minnesota, Minneapolis, MN 55455 USA. proven to be a more complex and generic problem [5]. For nonlinear oscillators with networked bidirectional coupling, such synchronization problems become more complex due to uncertainties introduced via networked communication channels. For example, individual systems could be connected via sampled-data coupling, thereby increasing the complexity of the associated synchronization problem [6], [7], [8].

Synchronization problems in sampled-data systems have been studied in recent years, and different approaches have been proposed to study the relation between sampling period, coupling strength, and synchronization properties [9], [10]. In existing results, it is typically considered that individual systems have the same sampling frequency, which is either constant or time-varying [8], [7]. However, in realistic settings, individual systems usually transmit information at different frequencies over a network, depending upon the communication channel, data traffic, etc. In this work, we consider a bidirectionally coupled, sampled-data, two-agent nonlinear system, wherein individual systems transmit information over a networked communication channel, asynchronously at possibly different (time-varying and uncertain) sampling intervals.

The main contribution of this paper is a novel approach towards synchronization analysis of nonlinear oscillatory systems, bidirectionally coupled via aperiodic sampled-data communication. By using tools based on input-output methods and dissipativity theory previously used to analyse stability of sampled-data systems [11], we provide a novel criterion that guarantees exponential synchronization of a twoagent nonlinear system, bidirectionally coupled via sampleddata synchronizing laws. In practical scenarios, it is desirable to have a certain measure of system performance. In synchronization problems, this implies achieving a specific transient rate of synchronization, which also depends on the oscillator dynamics. The result provided in this paper also takes the rate of synchronization into account, and aids in computing the trade-offs between coupling gain, rate of synchronization, and the maximum bound on the (time-varying, uncertain) sampling intervals.

The remainder of this paper has been structured as follows. In Section II, we introduce the problem setting under consideration, which consists of a two-agent nonlinear system, bidirectionally coupled via sampled-data coupling laws. In Section II-A, we introduce assumptions and existing results guaranteeing synchronization in the absence of sampled-data effects. In Section III, we remodel the system under consideration as a feedback-interconnection between a continuoustime system, and an operator that captures the sampled- 


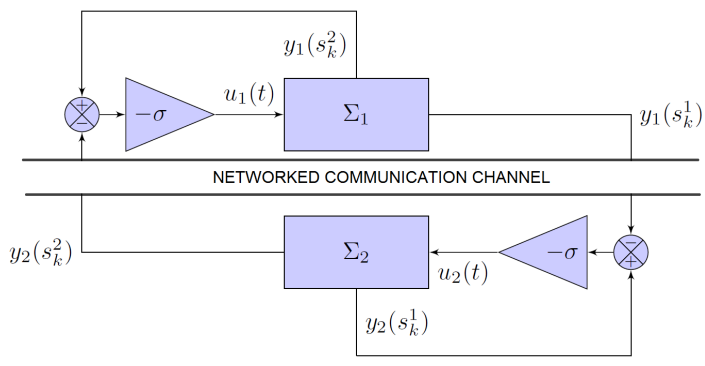

Fig. 1: Bidirectionally coupled agents $\Sigma_{1}$ and $\Sigma_{2}$ under (asynchronous) sampled-data transmission.

data/network effects. In Section IV, we provide the main result of this paper, where we use dissipativity theory based tools to study the feedback interconnection introduced in Section III, and provide a novel criterion that guarantees exponential synchronization. In Section $\mathrm{V}$, we provide a numerical example illustrating the application of our result.

Notation: $\mathbb{R}$ is the set of all real numbers, implying $\mathbb{R}^{n}$ is the set of all $n$-dimensional real vectors. The set of all natural numbers is denoted by $\mathbb{N}$. For an integer $n \geq 1, I_{n}$ denotes the $n \times n$ unit matrix. The Euclidean norm of a vector $z \in \mathbb{R}^{n}$ is denoted by $\|z\|=\sqrt{z^{T} z}$, where $z^{T}$ denotes the transposition of $z$.

\section{Problem Statement}

In this paper, we consider a 2-agent interconnected system as shown in Figure 1. Let the individual sub-system dynamics be given by

$$
\begin{aligned}
& \dot{x}_{i}(t)=f\left(x_{i}(t)\right)+B u_{i}(t), \\
& y_{i}(t)=C x_{i}(t), \quad i=1,2,
\end{aligned}
$$

where $x_{i} \in \mathbb{R}^{n}, u_{i}, y_{i} \in \mathbb{R}^{m}$ are the state, input, and output, of the $i^{t h}$ system, respectively. The function $f: \mathbb{R}^{n} \rightarrow \mathbb{R}^{n}$ is a sufficiently smooth vector field, and $B$ and $C$ are matrices with appropriate dimensions, with $C B=: b \in \mathbb{R}^{m \times m}$ and $b$ being positive definite and, without loss of generality, diagonal. This implies the system is considered to be of relative degree one. The output of the $i^{t h}$ sub-system is transmitted to the $j^{\text {th }}$ sub-system only at instants given by the sampling sequence

$$
s_{k+1}^{i}=s_{k}^{i}+h_{k}^{i}, h_{k}^{i} \in\left[\underline{h}_{i}, \bar{h}_{i}\right], k \in \mathbb{N},
$$

where $0 \leq \underline{h}_{i} \leq \bar{h}_{i}$. Without loss of generality, we consider $s_{0}^{i}=0, i=1,2$.

Assumption 1: The $i^{t h}$ sub-system has access to local output information at time instants $t=s_{k}^{j}, j \neq i, k \in \mathbb{N}$.

The aforementioned assumption represents scenarios in which individual sub-systems could be synchronously sampling at identical high frequencies, but transmit information asynchronously at different (and time-varying) frequencies, due to constraints imposed by the communication network. The local information, which is not transmitted over the network, can then be considered to be instantaneously available locally, and can hence be used for local feedback, when the output of the other system is received over the network. A second scenario justifying Assumption 1 is that local measurements could be performed in an event-based fashion, as soon as the sampled output of the other system is received. Under Assumption 1, the coupling laws can be expressed as follows:

$$
\begin{aligned}
& u_{1}(t)=-\sigma\left(y_{1}\left(s_{k}^{2}\right)-y_{2}\left(s_{k}^{2}\right)\right), \forall t \in\left[s_{k}^{2}, s_{k+1}^{2}\right), \\
& u_{2}(t)=-\sigma\left(y_{2}\left(s_{k}^{1}\right)-y_{1}\left(s_{k}^{1}\right)\right), \forall t \in\left[s_{k}^{1}, s_{k+1}^{1}\right),
\end{aligned}
$$

where $\sigma \in \mathbb{R}_{+}$is the constant coupling strength. We care to stress that Assumption 1 is key in making exact synchronization feasible. This is evident when we discuss the role of this assumption in the positive invariance of the synchronization manifold in the next sub-section. In the absence of such an assumption, typically, only approximate synchronization is feasible.

Definition 2: The bidirectionally coupled system given by (1), (3) is said to exponentially synchronize if

$$
\left\|e_{x}(t)\right\| \leq \rho e^{-\alpha t}\left\|e_{x}(0)\right\|, \forall t \geq 0
$$

where $\rho, \alpha>0, e_{x}(0) \in \mathbb{R}^{n}$, and $e_{x}(t):=x_{1}(t)-x_{2}(t)$ is the state synchronization error.

The goal of this paper is to analyse the exponential synchronization of system (1), (3).

\section{A. Assumptions and Preliminary Results}

In this section, in order to establish exponential synchronization of the coupled system (1), (3), in the sense of Definition 2, we introduce a few assumptions.

Assumption 3: The solutions of the closed-loop system (5), (3) are ultimately bounded.

Since the individual systems under consideration are of relative degree one, we know that there exists a well-defined coordinate transformation $z_{i}=\Phi\left(x_{i}\right), z_{i} \in \mathbb{R}^{n-m}, i=1,2$, such that the $i^{\text {th }}$ sub-system dynamics are given by

$$
\begin{aligned}
& \dot{z}_{i}(t)=q\left(z_{i}(t), y_{i}(t)\right), \\
& \dot{y}_{i}(t)=a\left(z_{i}(t), y_{i}(t)\right)+b u_{i}(t), i=1,2,
\end{aligned}
$$

where $z_{i} \in \mathbb{R}^{n-m}, u_{i}, y_{i} \in \mathbb{R}^{m}, q: \mathbb{R}^{n-m} \times \mathbb{R}^{m} \rightarrow \mathbb{R}^{n-m}$, and $a: \mathbb{R}^{n-m} \times \mathbb{R}^{m} \rightarrow \mathbb{R}^{m}$ [5]. In this paper, we will analyse the exponential synchronization of system (5), (3), which implies the exponential synchronization of system (1), (3). The remainder of this paper deals with system (5), (3), and all the results provided for this system, hold for system (1), (3) as well.

First, we introduce a definition of the convergence property, which will be exploited later to ensure certain synchronization properties.

Definition 4: [12] The dynamics $\dot{x}=g(x, u(t))$, with $u(t)$ a bounded time-varying input, are said to be convergent if

- there exists a solution $\bar{x}(t)$ which is bounded for all $t \in \mathbb{R}$

- $\bar{x}(t)$ is a globally asymptotically stable solution.

The solution $\bar{x}(t)$ is called the steady-state solution. If $\bar{x}(t)$ is in addition exponentially stable, then the system is called exponentially convergent.

Next, we consider the following assumption guaranteeing exponential convergence of internal dynamics of (5). 
Assumption 5: (Demidovich Condition [13]) There exists a positive definite matrix $P_{z} \in \mathbb{R}^{(n-m) \times(n-m)}$, such that the internal state dynamics given by $\dot{z}_{i}(t)=q\left(z_{i}(t), y_{i}(t)\right)$, $i=1,2$, satisfies

$$
P_{z} \frac{\partial q}{\partial z_{i}}\left(z_{i}, y_{i}\right)+\frac{\partial q^{T}}{\partial z_{i}}\left(z_{i}, y_{i}\right) P_{z} \leq-\delta I_{n-m}
$$

where $P_{z}=P_{z}^{T}>0$, and $\delta>0$.

Assumption 5 implies that the $z_{i}$-dynamics, for $i=1,2$, are exponentially convergent and establishes detectabilitytype conditions on individual sub-systems. This implies that on the synchronization manifold, when $y_{1}(t)=y_{2}(t) \equiv$ $y_{s}(t)$, the internal dynamics $\dot{z}_{i}(t)=q\left(z_{i}(t), y_{i}(t)\right), i=1,2$, converge to a unique, bounded solution only dependent on $y_{s}(t)$ and not on the (typically different) initial conditions of the sub-systems (see [14], Theorem 2.29).

In the absence of sampling, the corresponding ideal synchronizing input is given by

$$
\begin{aligned}
& u_{1}^{\star}(t)=-\sigma\left(y_{1}(t)-y_{2}(t)\right), \\
& u_{2}^{\star}(t)=-\sigma\left(y_{2}(t)-y_{1}(t)\right), \forall t \geq 0 .
\end{aligned}
$$

Now, we introduce a preliminary result on the bidirectionally coupled system (5), in the absence of sampling, i.e., with $u_{i}(t)=u_{i}^{\star}(t)$, given by the coupling law in (7).

Theorem 6: [5] Consider system (5) with $u_{i}(t)=u_{i}^{\star}(t)$ given by (7), and let Assumption 3 hold for system (5), with $u_{i}(t)=u_{i}^{\star}(t)$, and let Assumption 5 hold. Then, there exists a constant $\bar{\sigma}$ such that for all $\sigma>\bar{\sigma}$, the bidirectionally coupled system given by (5) with $u_{i}(t)=u_{i}^{\star}(t)$, achieves exponential synchronization.

Under the conditions of Theorem 6, there exists a blockdiagonal matrix $P_{y}=P_{y}^{T}>0$, such that the storage function

$$
V(e(t))=\left[\begin{array}{l}
y_{1}(t)-y_{2}(t) \\
z_{1}(t)-z_{2}(t)
\end{array}\right]^{T}\left[\begin{array}{cc}
P_{y} & 0 \\
0 & P_{z}
\end{array}\right]\left[\begin{array}{l}
y_{1}(t)-y_{2}(t) \\
z_{1}(t)-z_{2}(t)
\end{array}\right]
$$

characterizes the exponential stability properties of the synchronization manifold. This implies that using such a storage function, it can be shown that $\dot{V}+\alpha V \leq 0$, where $\alpha>0$ [5]. While the matrix $P_{z}$ results from Assumption 5, it has previously been shown that with the simple choice of $P_{y}=$ $I_{m}$, a candidate storage function of the form given in (8), can be used to characterize the exponential stability properties of the synchronization manifold (see [5], proof of Theorem 1). Later, we will exploit such type of storage function as a candidate storage function to show synchronization for the case with sampled-data coupling.

In the following lemma, we establish positive invariance of the synchronization manifold for system (5), (3), i.e., for the system with sampled-data coupling.

Lemma 7: The synchronization manifold given by

$$
\begin{aligned}
\mathcal{M}=\left\{\operatorname{col}\left(z_{1}, z_{2}, y_{1}, y_{2}\right) \in \mathbb{R}^{2 n}\right. \\
\left.: z_{1}=z_{2}, y_{1}=y_{2}\right\}
\end{aligned}
$$

is positively invariant under the bidirectionally coupled system (5), with $u_{i}(t), i \in\{1,2\}$ given by (3).

Proof: From (7), we know that on the synchronization manifold, $u_{1}(t)=u_{2}(t)=0$ for all $t \in \mathbb{R}$, since $y_{1}(t)=$ $y_{2}(t)$. Consequently, from (5), since on the synchronization manifold $\mathcal{M}, y_{1}(t)=y_{2}(t)$ and $z_{1}(t)=z_{2}(t)$, we have that $\dot{y}_{1}(t)=\dot{y}_{2}(t)$ and $\dot{z}_{1}(t)=\dot{z}_{2}(t)$. Therefore, we have that $\mathcal{M}$ is positively invariant.

Given the positive invariance of the synchronization manifold, we proceed to analyse the exponential synchronization of system (5), (3), which relates to the exponential stability properties of this manifold. In order to do so, in the next section, we will consider the effects of sampling as perturbations to the continuous-time networked system given by (5), with $u_{i}(t)=u_{i}^{\star}(t)$ in (7).

\section{SyStEM REFORMULATION}

In this section, we remodel the networked system, given by (5), (3), as the feedback interconnection (shown in Figure 2) of a system operator $\mathbf{G}$ representing the dynamics of the networked system with continuous-time coupling, i.e., with $u_{i}(t)=u_{i}^{\star}(t)$ given by (7), and an operator $\boldsymbol{\Delta}$ that captures the error introduced by asynchronous sampling.

Consider an operator $\Delta_{i}: \mathbb{R}^{m} \mapsto \mathbb{R}^{m}$, such that for any signal $\rho$, it follows that

$$
\left(\Delta_{i} \rho\right)(t)=-\int_{s_{k}^{i}}^{t} \rho(s) d s, \forall t \in\left[s_{k}^{i}, s_{k+1}^{i}\right), k \in \mathbb{N}, i=1,2 .
$$

In the following lemma, we will show how the sampleddata synchronizing input (3) is equivalent to the ideal continuous-time synchronizing input (7), subject to a sampling-induced perturbation captured by the operator $\Delta_{i}$.

Lemma 8: Consider the operator $\Delta_{i}$ given in (10). Then, the sampled-data coupling law given by (3) can be expressed as

$$
u_{i}(t)=u_{i}^{\star}(t)+\sigma \Delta_{j}\left(\dot{y}_{j}-\dot{y}_{i}\right)(t), i=1,2, j \neq i
$$

where $u_{i}^{\star}(t)$ is given by (7).

Proof: Recalling the definition of the sampled-data synchronizing input in (3), we have, for $i=1,2$,

$$
\begin{aligned}
u_{i}(t)= & -\sigma\left(y_{i}\left(s_{k}^{j}\right)-y_{j}\left(s_{k}^{j}\right)\right), \forall t \in\left[s_{k}^{j}, s_{k+1}^{j}\right), j \neq i \\
= & -\sigma\left(y_{i}\left(s_{k}^{j}\right)-y_{j}\left(s_{k}^{j}\right)+y_{i}(t)-y_{i}(t)\right. \\
\quad & \left.\quad y_{j}(t)-y_{j}(t)\right) \\
= & -\sigma\left(\left(y_{i}(t)-y_{j}(t)\right)+\left(y_{j}(t)-y_{j}\left(s_{k}^{j}\right)\right)\right. \\
& \left.\quad\left(y_{i}(t)-y_{i}\left(s_{k}^{j}\right)\right)\right) .
\end{aligned}
$$

From (7), we know that, for $i=1,2, j \neq i$, the term $-\sigma\left(y_{i}(t)-y_{j}(t)\right)$ in (12) is the $i^{t h}$ synchronizing input without sampling, denoted by $u_{i}^{\star}(t)$. Consequently,

$$
u_{i}(t)=u_{i}^{\star}(t)+\sigma\left(y_{j}\left(s_{k}^{j}\right)-y_{j}(t)\right)-\sigma\left(y_{i}\left(s_{k}^{j}\right)-y_{i}(t)\right) .
$$

Using the definition of operator $\Delta_{i}$ in (10), we obtain

$$
\begin{aligned}
u_{i}(t) & =u_{i}^{\star}(t)+\sigma\left(\Delta_{j} \dot{y}_{j}\right)(t)-\sigma\left(\Delta_{j} \dot{y}_{i}\right)(t), \\
& =u_{i}^{\star}(t)+\sigma \Delta_{j}\left(\dot{y}_{j}-\dot{y}_{i}\right)(t) .
\end{aligned}
$$


Based on Lemma 8, we now proceed to remodel the bidirectionally coupled system (5), (3). In order to do so, we define the output synchronization error by

$$
e_{y}(t)=y_{1}(t)-y_{2}(t)
$$

and the internal dynamics synchronization error by

$$
e_{z}(t)=z_{1}(t)-z_{2}(t) \text {. }
$$

Using the aforementioned notations, in the following lemma, we will formulate the synchronization error dynamics for system (5), (3). The stability property of these error dynamics are directly related to those of the synchronization manifold and hence will be investigated later (in Section IV) to formulate conditions for synchronization.

Lemma 9: Consider a system operator $\mathbf{G}$, representing the synchronization error dynamics given by

$$
\begin{aligned}
\dot{e}_{y}(t)= & a\left(e_{z}(t)+z_{2}(t), e_{y}(t)+y_{2}(t)\right)-a\left(z_{2}(t), y_{2}(t)\right) \\
& -2 b \sigma e_{y}(t)+b \sigma w(t), \\
\dot{e}_{z}(t)= & q\left(e_{z}(t)+z_{2}(t), e_{y}(t)+y_{2}(t)\right)-q\left(z_{2}(t), y_{2}(t)\right),
\end{aligned}
$$

and the operator $\Delta$ such that

$$
\begin{aligned}
w(t) & :=\left(\boldsymbol{\Delta} \dot{e}_{y}\right)(t):=-\left(\Delta_{2} \dot{e}_{y}\right)(t)-\left(\Delta_{1} \dot{e}_{y}\right)(t), \\
& =w_{1}(t)+w_{2}(t),
\end{aligned}
$$

where $w_{1}(t):=-\left(\Delta_{2} \dot{e}_{y}\right)(t)$ and $w_{2}(t):=-\left(\Delta_{1} \dot{e}_{y}\right)(t)$, with $\Delta_{i}, i \in\{1,2\}$, given by (10). Then, the feedback interconnection of $\mathbf{G}$ and $\boldsymbol{\Delta}$, given by (17), (18), represents the synchronization error dynamics of the bidirectionally coupled system (5), (3).

Proof: Consider the output synchronization error in (15). We have,

$$
\dot{e}_{y}(t)=\dot{y}_{1}(t)-\dot{y}_{2}(t) \text {. }
$$

Using the definition of $\dot{y}_{i}(t), i=1,2$ in (5), we have

$$
\dot{e}_{y}(t)=a\left(z_{1}(t), y_{1}(t)\right)+b u_{1}(t)-a\left(z_{2}(t), y_{2}(t)\right)-b u_{2}(t) \text {. }
$$

From Lemma 8, using the reformulation of $u_{1}(t)$ and $u_{2}(t)$, given by (11), we obtain

$$
\begin{aligned}
\dot{e}_{y}(t)= & a\left(z_{1}(t), y_{1}(t)\right)-a\left(z_{2}(t), y_{2}(t)\right) \\
& +b u_{1}^{\star}(t)+b \sigma \Delta_{2}\left(\dot{y}_{2}-\dot{y}_{1}\right)(t) \\
& -b u_{2}^{\star}(t)-b \sigma \Delta_{1}\left(\dot{y}_{1}-\dot{y}_{2}\right)(t) .
\end{aligned}
$$

Recalling the definition of $u_{1}^{\star}(t)$ and $u_{2}^{\star}(t)$ in (7), and the definition of $e_{y}$ in (15), it holds that

$$
\begin{aligned}
& u_{1}^{\star}(t)=-\sigma e_{y}(t), \\
& u_{2}^{\star}(t)=\sigma e_{y}(t) .
\end{aligned}
$$

Consequently, from (21), we have that

$$
\begin{aligned}
\dot{e}_{y}(t)= & a\left(z_{1}(t), y_{1}(t)\right)-a\left(z_{2}(t), y_{2}(t)\right) \\
& -b \sigma e_{y}(t)+b \sigma \Delta_{2}\left(\dot{y}_{2}-\dot{y}_{1}\right)(t) \\
& -b \sigma e_{y}(t)-b \sigma \Delta_{1}\left(\dot{y}_{1}-\dot{y}_{2}\right)(t), \\
= & a\left(e_{z}(t)+z_{2}(t), e_{y}(t)+y_{2}(t)\right)-a\left(z_{2}(t), y_{2}(t)\right) \\
& -2 b \sigma e_{y}(t)+b \sigma\left(\Delta_{2}\left(-\dot{e}_{y}\right)(t)-\Delta_{1}\left(\dot{e}_{y}\right)(t)\right)
\end{aligned}
$$

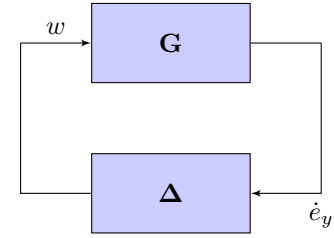

Fig. 2: The feedback interconnection of $\mathbf{G}$ and $\boldsymbol{\Delta}$, representing the system (5), (3).

Now, recalling the internal dynamics synchronization error defined in (16), we get

$$
\begin{aligned}
\dot{e}_{z}(t) & =\dot{z}_{1}(t)-\dot{z}_{2}(t) \\
& =q\left(z_{1}(t), y_{1}(t)\right)-q\left(z_{2}(t), y_{2}(t)\right) \\
& =q\left(e_{z}(t)+z_{2}(t), e_{y}(t)+y_{2}(t)\right)-q\left(z_{2}(t), y_{2}(t)\right),
\end{aligned}
$$

Therefore, the synchronization error dynamics of system (5), (3) can be expressed using a system operator $\mathbf{G}$ (see Figure 2), defined by the error dynamics

$$
\begin{aligned}
\dot{e}_{y}(t)= & a\left(e_{z}(t)+z_{2}(t), e_{y}(t)+y_{2}(t)\right)-a\left(z_{2}(t), y_{2}(t)\right) \\
& -2 b \sigma e_{y}(t)+b \sigma w(t), \\
\dot{e}_{z}(t)= & q\left(e_{z}(t)+z_{2}(t), e_{y}(t)+y_{2}(t)\right)-q\left(z_{2}(t), y_{2}(t)\right),
\end{aligned}
$$

where $w(t)$ is a perturbation induced by the error due to asynchronous sampling and is defined by the operator $\Delta$ according to

$$
\begin{aligned}
w(t) & =\left(\boldsymbol{\Delta} \dot{e}_{y}\right)(t):=-\left(\Delta_{2} \dot{e}_{y}\right)(t)-\left(\Delta_{1} \dot{e}_{y}\right)(t), \\
& =w_{1}(t)+w_{2}(t),
\end{aligned}
$$

where $w_{1}(t):=-\left(\Delta_{2} \dot{e}_{y}\right)(t)$ and $w_{2}(t):=-\left(\Delta_{1} \dot{e}_{y}\right)(t)$.

\section{Main Result}

In this section, we provide conditions that guarantee exponential synchronization of the bidirectionally coupled systems (5), (3). First, we provide a generic result that holds for the feedback-interconnection $G-\Delta$. By studying the properties of the feedback-interconnection $\mathbf{G}-\boldsymbol{\Delta}$, we provide conditions that guarantee exponential stability of the origin of the error dynamics concerning $e_{y}(t)$ and $e_{z}(t)$, i.e., exponential synchronization of the system (5), (3).

Theorem 10: Consider the feedback interconnection $\mathbf{G}-$ $\Delta$ given by (17), (18), and assume that

1) there exists a supply function $\mathcal{S}: \mathbb{R}^{m} \times \mathbb{R}^{m} \mapsto \mathbb{R}$ so that

$$
\int_{0}^{t} \mathcal{S}\left(\theta, \dot{e}_{y}(\theta), w_{1}(\theta), w_{2}(\theta)\right) d \theta \leq 0, \forall t \geq 0,
$$

where $\dot{e}_{y}$ is given by (17), and $w_{1}, w_{2}$ are defined below (18).

2) there exists a continuously differentiable storage function $V: \mathbb{R}^{n} \rightarrow \mathbb{R}^{+}$and scalars $0<c_{1}<c_{2}, \alpha>0$ such that

$$
c_{1}\|e\|^{2} \leq V(e) \leq c_{2}\|e\|^{2},
$$

and

$$
\dot{V}(e(t))+\alpha V(e(t)) \leq e^{-\alpha t} \mathcal{S}\left(t, \dot{e}_{y}(t), w(t)\right), \forall t \geq 0,
$$


where $e(t)=\left[\begin{array}{ll}e_{y}^{T}(t) & e_{z}^{T}(t)\end{array}\right]^{T}$ is governed by (17). Then, the equilibrium $e=0$ of system (17), (18) is globally exponentially stable with a decay rate of at least $\alpha / 2$.

Proof: Consider the function

$$
\begin{aligned}
W(t)= & e^{\alpha t} V(e(t)) \\
& \quad-\int_{0}^{t} \mathcal{S}\left(\theta, \dot{e}_{y}(\theta), w_{1}(\theta), w_{2}(\theta)\right) d \theta, \forall t \geq 0 .
\end{aligned}
$$

From condition (29), we can conclude $\dot{W}(t) \leq 0, \forall t \geq 0$, implying $W(t) \leq W(0)$, i.e.,

$$
e^{\alpha t} V(e(t))-\int_{0}^{t} \mathcal{S}\left(\theta, \dot{e}_{y}(\theta), w_{1}(\theta), w_{2}(\theta)\right) d \theta \leq V(e(0)) .
$$

Therefore,

$$
e^{\alpha t} V(e(t)) \leq V(e(0))+\int_{0}^{t} \mathcal{S}\left(\theta, \dot{e}_{y}(\theta), w_{1}(\theta), w_{2}(\theta)\right) d \theta
$$

and using condition (27), we have

$$
V(e(t)) \leq e^{-\alpha t} V(e(0)) .
$$

Consequently, using (28), we obtain

$$
\|e(t)\|^{2} \leq \frac{c_{2}}{c_{1}} e^{-\alpha t}\|e(0)\|^{2},
$$

i.e.,

$$
\|e(t)\| \leq \sqrt{\frac{c_{2}}{c_{1}}} e^{\frac{-\alpha}{2} t}\|e(0)\|,
$$

implying that the equilibrium $e=0$, of system (17), (18) is exponentially stable with a decay rate of at least $\alpha / 2$.

Theorem 10 holds for any operator $\Delta$, as long as its properties can be characterized using a supply function satisfying (27). In the case of sampling-induced perturbations captured by the operator $\Delta$, as defined in (18), we characterize the properties of the operator $\boldsymbol{\Delta}$ as shown in the following lemma.

Lemma 11: Consider the operator $\Delta$ defined in (18), (10). Then, for any $R_{1} \in \mathbb{R}^{m \times m}$ and $R_{2} \in \mathbb{R}^{m \times m}$, with $R_{1}=R_{1}^{T}>0$ and $R_{2}=R_{2}^{T}>0, \Delta$ satisfies

$$
\int_{0}^{t} S\left(\theta, \dot{e}_{y}(\theta), w_{1}(\theta), w_{2}(\theta)\right) \leq 0, \forall t \geq 0,
$$

where $\alpha>0$, and the function $S: \mathbb{R}^{+} \times \mathbb{R}^{m} \times \mathbb{R}^{m} \mapsto \mathbb{R}$ is given by

$$
\begin{aligned}
\mathcal{S}\left(t, \dot{e}_{y}(t), w_{1}(t), w_{2}(t)\right) & \\
= & e^{\alpha t}\left(\left[\begin{array}{c}
w_{1}(t) \\
w_{2}(t)
\end{array}\right]^{T}\left[\begin{array}{cc}
R_{1} & 0 \\
0 & R_{2}
\end{array}\right]\left[\begin{array}{l}
w_{1}(t) \\
w_{2}(t)
\end{array}\right]\right. \\
& \left.-\dot{e}_{y}^{T}(t)\left(\bar{h}_{2}^{2} e^{\alpha \bar{h}_{2}} R_{1}+\bar{h}_{1}^{2} e^{\alpha \bar{h}_{1}} R_{2}\right) \dot{e}_{y}(t)\right),
\end{aligned}
$$

with $\bar{h}_{1}, \bar{h}_{2}$ given by (2), and $w_{1}(t), w_{2}(t)$ defined by (26).

Proof: Consider the term $w_{1}(t)$ in (18), and the definition of operator $\Delta_{2}$ from (10). We have,

$$
\begin{aligned}
w_{1}(t) & =-\int_{s_{k}^{2}}^{t} \dot{e}_{y}(s) d s . \forall t \in\left[s_{k}^{2}, s_{k+1}^{2}\right), k \in \mathbb{N}, \\
& =e_{y}\left(s_{k}^{2}\right)-e_{y}(t) .
\end{aligned}
$$

Using Jensen's inequality [15], we obtain

$$
\begin{aligned}
w_{1}^{T}(t) R_{1} w_{1}(t) & \leq\left(t-s_{k}^{2}\right) \int_{s_{k}^{2}}^{t} \dot{e}_{y}^{T}(\zeta) R_{1} \dot{e}_{y}(\zeta) d \zeta \\
& \leq \bar{h}_{2} \int_{s_{k}^{2}}^{t} \dot{e}_{y}^{T}(\zeta) R_{1} \dot{e}_{y}(\zeta) d \zeta .
\end{aligned}
$$

Using the change of variable $s=\zeta-t$, we obtain

$$
\begin{aligned}
w_{1}^{T}(t) R_{1} w_{1}(t) & \leq \bar{h}_{2} \int_{s_{k}^{2}-t}^{0} \dot{e}_{y}^{T}(t+s) R_{1} \dot{e}_{y}(t+s) d s \\
& \leq \bar{h}_{2} \int_{-\bar{h}_{2}}^{0} \dot{e}_{y}^{T}(t+s) R_{1} \dot{e}_{y}(t+s) d s .
\end{aligned}
$$

Therefore,

$$
\begin{aligned}
\int_{0}^{t} e^{\alpha \theta} w_{1}^{T}(\theta) R_{1} w_{1}(\theta) d \theta \leq \\
\bar{h}_{2} \int_{0}^{t} e^{\alpha \theta}\left(\int_{-\bar{h}_{2}}^{0} \dot{e}_{y}^{T}(\theta+s) R_{1} \dot{e}_{y}(\theta+s) d s\right) d \theta .
\end{aligned}
$$

Substituting $u=\theta+s$, we have that

$$
\begin{aligned}
& \int_{0}^{t} e^{\alpha \theta} w_{1}^{T}(\theta) R_{1} w_{1}(\theta) d \theta \\
& \leq \bar{h}_{2} \int_{-\bar{h}_{2}}^{0}\left(\int_{s}^{t+s} e^{\alpha(u-s)} \dot{e}_{y}^{T}(u) R_{1} \dot{e}_{y}(u) d u\right) d s .
\end{aligned}
$$

Since the inner integral in the right-hand side of the inequality in (42) is always positive because $R_{1}$ is positive definite, we can upper bound the left-hand side in (42) using the limits of $s$ and obtain

$$
\begin{aligned}
\int_{0}^{t} e^{\alpha \theta} & w_{1}^{T}(\theta) R_{1} w_{1}(\theta) d \theta \\
& \leq \bar{h}_{2} \int_{-\bar{h}_{2}}^{0}\left(\int_{-\bar{h}_{2}}^{t+0} e^{\alpha\left(u+\bar{h}_{2}\right)} \dot{e}_{y}^{T}(u) R_{1} \dot{e}_{y}(u) d u\right) d s \\
& =\bar{h}_{2} e^{\alpha \bar{h}_{2}} \int_{-\bar{h}_{2}}^{0}\left(\int_{0}^{t} e^{\alpha u} \dot{e}_{y}^{T}(u) R_{1} \dot{e}_{y}(u) d u\right) d s \\
& =\bar{h}_{2}^{2} e^{\alpha \bar{h}_{2}} \int_{0}^{t} e^{\alpha \theta} \dot{e}_{y}^{T}(\theta) R_{1} \dot{e}_{y}(\theta) d \theta .
\end{aligned}
$$

Similarly, for the term $w_{2}(t)=-\left(\Delta_{1} \dot{e}_{y}\right)(t)$, we can obtain

$$
\begin{aligned}
\int_{0}^{t} e^{\alpha \theta} & w_{2}^{T}(\theta) R_{2} w_{2}(\theta) d \theta \\
\quad \leq & \bar{h}_{1}^{2} e^{\alpha \bar{h}_{1}} \int_{0}^{t} e^{\alpha \theta} \dot{e}_{y}^{T}(\theta) R_{2} \dot{e}_{y}(\theta) d \theta
\end{aligned}
$$

Summation of (43) and (44) gives

$$
\int_{0}^{t} \mathcal{S}\left(\theta, \dot{e}_{y}(\theta), w(\theta)\right) \leq 0
$$

where

$$
\begin{aligned}
\mathcal{S}\left(t, \dot{e}_{y}(t), w_{1}(t), w_{2}(t)\right) & \\
= & e^{\alpha t}\left(\left[\begin{array}{c}
w_{1}(t) \\
w_{2}(t)
\end{array}\right]^{T}\left[\begin{array}{cc}
R_{1} & 0 \\
0 & R_{2}
\end{array}\right]\left[\begin{array}{l}
w_{1}(t) \\
w_{2}(t)
\end{array}\right]\right. \\
& \left.-\dot{e}_{y}^{T}(t)\left(\bar{h}_{2}^{2} e^{\alpha \bar{h}_{2}} R_{1}+\bar{h}_{1}^{2} e^{\alpha \bar{h}_{1}} R_{2}\right) \dot{e}_{y}(t)\right) .
\end{aligned}
$$

This concludes the proof.

Remark: The aforementioned lemma is an extension of the result provided in [11], wherein an operator was used to capture the effects of sampling in a single-loop input-affine nonlinear system.

We now proceed to provide a dissipativity-based criterion that guarantees exponential synchronization of the system (5), (3), by using the supply function given in Lemma 11, in conjunction with the result given in Theorem 10. 
Theorem 12: Consider the bidirectionally coupled system (5), (3) satisfying Assumption 3 and Assumption 5, the feedback-interconnection (17), (18), the supply function in (37), and the storage function $V: \mathbb{R}^{n} \rightarrow \mathbb{R}^{+}$of the form given in (8). Then, for a given coupling gain $\sigma$ and sampling interval bounds $\bar{h}_{1}, \bar{h}_{2}$, the system (5), (3) exponentially synchronizes with a decay-rate of at least $\alpha / 2$, if there exist matrices $P_{y}=P_{y}^{T}>0, P_{z}=P_{z}^{T}>0, R_{1}=R_{1}^{T}>0$ and $R_{2}=R_{2}^{T}>0$ such that

$$
\begin{aligned}
& \dot{e}_{y}^{T} P_{y} e_{y}+\dot{e}_{z}^{T} P_{z} e_{z}+e_{y}^{T} P_{y} \dot{e}_{y}+e_{z}^{T} P_{z} \dot{e}_{z}+\alpha\left(e_{y}^{T} P_{y} e_{y}\right. \\
& \left.+e_{z}^{T} P_{z} e_{z}\right)-w_{1}^{T} R_{1} w_{1}-w_{2}^{T} R_{2} w_{2}+\dot{e}_{y}^{T}\left(\bar{h}_{2}^{2} e^{\alpha h_{2}} R_{1}\right. \\
& \left.\quad+\bar{h}_{1}^{2} e^{\alpha \bar{h}_{1}} R_{2}\right) \dot{e}_{y} \leq 0 .
\end{aligned}
$$

Proof: From the result given in Lemma 11, we know that the supply function in (37) satisfies condition (27) in Theorem 10.

Additionally, from Theorem 6, we know that the storage function $V$ in (8) characterizes the exponential stability properties of the synchronization manifold, in the absence of sampling-induced effects. Using the block-diagonal structure of this storage function, condition (28) in Theorem 10 can be easily shown.

Now, using the storage function in (8), and the supply function in (37), the inequality condition (29) in Theorem 10 , corresponds to (47).

Therefore, if condition (47) is satisfied for $\alpha>0$, and a given coupling gain $\sigma$ and sampling-interval bounds $\bar{h}_{1}, \bar{h}_{2}$, then, as a consequence of Theorem 10, we know that system (5), (3) exponentially synchronizes with a decay-rate of at least $\alpha / 2$.

Remark: As a result of Theorem 6, the storage function $V$ in (8), will satisfy inequality (29) in the absence of sampling, i.e., when $\mathcal{S}\left(\theta, \dot{e}_{y}(\theta), w_{1}(\theta), w_{2}(\theta)\right)=0$. Additionally, in the continuous-time case, the decay-rate $\alpha$ in (29) (with $\mathcal{S}\left(t, \dot{e}_{y}(t), w_{1}(t), w_{2}(t)\right)=0$ ) will depend on $P_{z}, P_{y}, \delta$ (in Assumption 5) and $\sigma$ (given $\sigma>\bar{\sigma}$ ).

In the next section, we will illustrate how condition (47) aids in deciding the trade-off between the sampling interval bounds $\bar{h}_{1}$ and $\bar{h}_{2}$, the coupling gain $\sigma$, and the exponential synchronization rate $\alpha / 2$.

\section{NumericAl EXAMPLE}

In this section, we consider a two-agent Fitzhugh-Nagumo system ([16], [17]) to illustrate the application of the result given in Theorem 10. The individual sub-system dynamics is given by

$$
\begin{aligned}
& \dot{y}_{i}=y_{i}-\frac{y_{i}^{3}}{3}-z_{i}+I+u_{i}, \\
& \dot{z}_{i}=\zeta\left(y_{i}+a-b z_{i}\right), i \in\{1,2\},
\end{aligned}
$$

where $I=0.5, a=0.7, b=0.8, \zeta=0.08$, and the synchronizing coupling laws are given by (3). Considering the error terms $e_{y}=y_{1}-y_{2}$, and $e_{z}=z_{1}-z_{2}$, the error dynamics are given by

$$
\begin{aligned}
\dot{e}_{y} & =\dot{y}_{1}-\dot{y}_{2} \\
& =e_{y}-e_{z}-2 \sigma e_{y}-\frac{y_{1}^{3}-y_{2}^{3}}{3},
\end{aligned}
$$

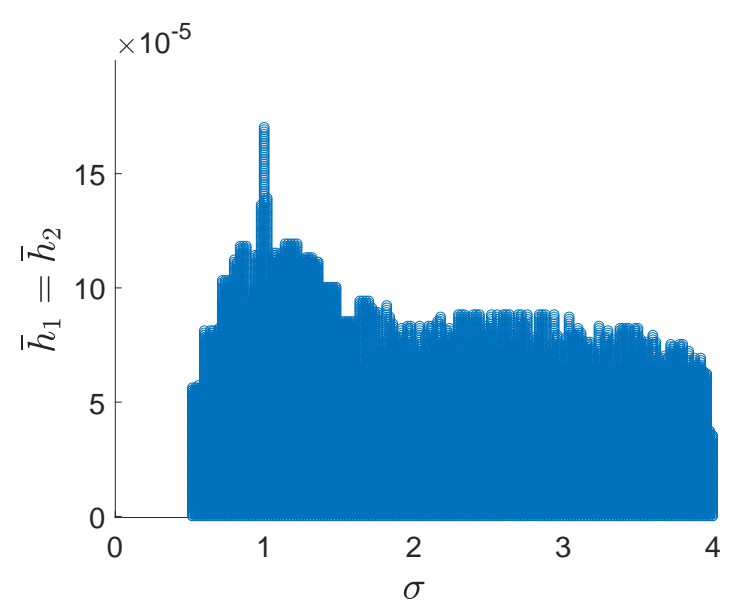

Fig. 3: Trade-off between coupling strength $\sigma$ and maximum sampling interval $\bar{h}_{1}=\bar{h}_{2}$, for the system (48), with $\alpha=$ 0.01 .

and

$$
\dot{e}_{z}=\zeta\left(e_{y}-b e_{z}\right)=0.08\left(e_{y}-e_{z}\right) .
$$

We know that by choosing the storage function $V$ in (8), and considering the supply function given in (37), inequality (29) leads to (47). For the system (48), using the definition of $\dot{e}_{y}$ in (49), and the fact that

$$
y_{1}^{3}-y_{2}^{3}=\frac{1}{4}\left(\left(y_{1}-y_{2}\right)^{3}+3\left(y_{1}+y_{2}\right)^{2}\left(y_{1}-y_{2}\right)\right),
$$

condition (47) corresponds to

$$
\begin{aligned}
& \left(e_{y}^{2}-e_{z} e_{y}-2 \sigma e_{y}^{2}-\frac{1}{12}\left(e_{y}^{4}+3\left(e_{y}+2 y_{2}\right)^{2} e_{y}^{2}\right)\right)\left(2 P_{y}\right. \\
& \left.+\bar{h}_{2}^{2} e^{\alpha \bar{h}_{2}} R_{1}+\bar{h}_{1}^{2} e^{\alpha \bar{h}_{1}} R_{2}\right)+2 P_{z} \zeta e_{z}\left(e_{y}-b e_{z}\right) \\
& +\alpha P_{y} e_{y}^{2}+\alpha P_{z} e_{z}^{2}-R_{1} w_{1}^{2}-R_{2} w_{2}^{2} \leq 0 .
\end{aligned}
$$

Additionally, the Demidovich condition introduced in Assumption 5 , leads to

$$
2 P_{z}(-\zeta b) \leq-\delta, \delta>0
$$

Inequality (52) can be used to analyse the trade-off between $\bar{h}_{1}, \bar{h}_{2}, \alpha$ and $\sigma$, for system (48). An exemplary feasibility region is computed using SOSTOOLS, by fixing $\alpha=0.01$, considering $\bar{h}_{1}=\bar{h}_{2}$, and solving inequalities (52) and (53). The synchronization diagram giving the tradeoff between coupling gain $\sigma$ and the maximum sampling bounds $\bar{h}_{1}=\bar{h}_{2}$, is given in Figure 3 . The profile of the synchronization diagram in Figure 3, is similar to the profile obtained for two Fitzhugh-Nagumo systems with time-delayed coupling [18]. When $\alpha=0.01$, the critical value of $\sigma$, below which synchronization is not possible, was found to be $\sigma=\bar{\sigma}=0.35$. In the absence of sampling, i.e., with $\bar{h}_{1}=\bar{h}_{2}=0$, as evident from Figure 3 , the obtained $\bar{\sigma}$ will be the same.

\section{CONCLUSION}

In this paper, a novel dissipativity-based approach towards synchronization analysis of nonlinear oscillatory systems with bidirectional sampled-data coupling, is provided. The approach builds on remodelling the sampled-data system 
as a feedback-interconnection of a continuous-time system operator that captures the bidirectionally coupled system dynamics in the absence of sampling, and an operator that captures the effects of sampling. The properties of this feedback-interconnection are then studied to provide dissipativity-based conditions that guarantee exponential synchronization of the nonlinear oscillatory systems with bidirectional sampled-data coupling. Finally, a two-agent Fitzhugh-Nagumo system is used to illustrate the theoretical results.

\section{REFERENCES}

[1] F. L. Lewis, H. Zhang, K. Hengster-Movric, and A. Das, Introduction to Synchronization in Nature and Physics and Cooperative Control for Multi-Agent Systems on Graphs. London: Springer London, 2014.

[2] H. Nijmeijer and I. M. Y. Mareels, "An observer looks at synchronization," IEEE Transactions on Circuits and Systems I: Fundamental Theory and Applications, vol. 44, no. 10, pp. 882-890, Oct 1997.

[3] F. Dörfler and F. Bullo, "Exploring synchronization in complex oscillator networks," in 51st IEEE Conference on Decision and Control (CDC), Dec 2012, pp. 7157-7170.

[4] J. Cosp, J. Madrenas, E. Alarcon, E. Vidal, and G. Villar, "Synchronization of nonlinear electronic oscillators for neural computation," IEEE Transactions on Neural Networks, vol. 15, no. 5, pp. 13151327, Sep. 2004.

[5] A. Y. Pogromsky, "Passivity based design of synchronizing systems," International Journal of Bifurcation and Chaos, vol. 08, no. 02, pp. 295-319, 1998.

[6] J. Lu and D. J. Hill, "Global asymptotical synchronization of chaotic Lur'e systems using sampled data: A linear matrix inequality approach," IEEE Transactions on Circuits and Systems II: Express Briefs, vol. 55, no. 6 , pp. 586-590, June 2008.

[7] T. H. Lee and J. H. Park, "Improved criteria for sampled-data synchronization of chaotic Lur'e systems using two new approaches," Nonlinear Analysis: Hybrid Systems, vol. 24, pp. 132 - 145, 2017.

[8] B. Shen, Z. Wang, and X. Liu, "Sampled-data synchronization control of dynamical networks with stochastic sampling," IEEE Transactions on Automatic Control, vol. 57, no. 10, pp. 2644-2650, Oct 2012.

[9] K. Sakai and T. Oguchi, "Synchronization of coupled nonlinear systems with bidirectional sampled-data couplings," IFAC-PapersOnLine, vol. 52, no. 16, pp. $634-639,2019,11$ th IFAC Symposium on Nonlinear Control Systems NOLCOS 2019.

[10] C. Hua, C. Ge, and X. Guan, "Synchronization of chaotic Lur'e systems with time delays using sampled-data control," IEEE Transactions on Neural Networks and Learning Systems, vol. 26, no. 6, pp. 12141221, June 2015.

[11] H. Omran, L. Hetel, M. Petreczky, J.-P. Richard, and F. LamnabhiLagarrigue, "Stability analysis of some classes of input-affine nonlinear systems with aperiodic sampled-data control," Automatica, vol. 70, pp. $266-274,2016$.

[12] B. P. Demidovich, "Lectures on the mathematical stability theory (in russian)," Nauka, Moscow, 1967.

[13] A. Pavlov, N. van de Wouw, and H. Nijmeijer, Convergent Systems: Analysis and Synthesis. Berlin, Heidelberg: Springer Berlin Heidelberg, 2005, pp. 131-146.

[14] A. Pavlov, N. van de Wouw, and H. Nijmeijer, Uniform output regulation of nonlinear systems : a convergent dynamics approach, ser. Systems and control : foundations and applications. Switzerland: Birkhäuser Verlag, 2006.

[15] K. Gu, J. Chen, and V. Kharitonov, Stability of Time-Delay Systems. Birkhauser Boston, 2003.

[16] R. FitzHugh, "Impulses and physiological states in theoretical models of nerve membrane," Biophysical Journal, vol. 1, no. 6, pp. 445 - 466, 1961.

[17] J. Nagumo, S. Arimoto, and S. Yoshizawa, "An active pulse transmission line simulating nerve axon," Proceedings of the IRE, vol. 50, no. 10, pp. 2061-2070, Oct 1962.

[18] E. Steur, W. Michiels, H. Huijberts, and H. Nijmeijer, "Networks of diffusively time-delay coupled systems: Conditions for synchronization and its relation to the network topology," Physica D: Nonlinear Phenomena, vol. 277, pp. 22 - 39, 2014. 